\title{
Diffusion Properties of Normal-Appearing White Matter Microstructure and Severity of Motor Impairment in Acute Ischemic Stroke
}

\author{
(D) C. Ingo, (D) C. Lin, (D). Higgins, (D) Y.A. Arevalo, and (D) S. Prabhakaran
}

\begin{abstract}
BACKGROUND AND PURPOSE: The effect of white matter hyperintensities as measured by FLAIR MR imaging on functional impairment and recovery after ischemic stroke has been investigated thoroughly. However, there has been growing interest in investigating normal-appearing white matter microstructural integrity following ischemic stroke onset with techniques such as DTI.

MATERIALS AND METHODS: Fifty-two patients with acute ischemic stroke and 36 without stroke were evaluated with a DTI and FLAIR imaging protocol and clinically assessed for the severity of motor impairment using the Motricity Index within 72 hours of suspected symptom onset.

RESULTS: There were widespread decreases in fractional anisotropy and increases in mean diffusivity and radial diffusivity for the acute stroke group compared with the nonstroke group. There was a significant positive association between fractional anisotropy and motor function and a significant negative association between mean diffusivity/radial diffusivity and motor function. The normal-appearing white matter ROls that were most sensitive to the Motricity Index were the anterior/posterior limb of the internal capsule in the infarcted hemisphere and the splenium of the corpus callosum, external capsule, posterior limb/ retrolenticular part of the internal capsule, superior longitudinal fasciculus, and cingulum (hippocampus) of the intrahemisphere/ contralateral hemisphere.
\end{abstract}

CONCLUSIONS: The microstructural integrity of normal-appearing white matter is a significant parameter to identify neural differences not only between those individuals with and without acute ischemic stroke but also correlated with the severity of acute motor impairment.

ABBREVIATIONS: $A D$ = axial diffusivity; $A D C=$ apparent diffusion coefficient; $D T I=$ diffusion tensor imaging; $D W I=$ diffusion-weighted imaging; $F A=$ fractional anisotropy; InfHem = infarcted hemisphere; IQR = interquartile range; NAWM = normal-appearing white matter; $M D=$ mean diffusivity; $R D=$ radial diffusivity; $\mathrm{WMH}=$ white matter hyperintensities

$\mathbf{T}$ he effect of white matter hyperintensities $(\mathrm{WMH})$ as measured by FLAIR MR imaging on functional impairment and recovery after ischemic stroke has been investigated thoroughly. ${ }^{1-7}$ However, there has been growing interest to investigate normalappearing white matter (NAWM) microstructural integrity, ${ }^{8,9}$ that is neither classified as infarct/edema/lesion tissue ${ }^{10,11}$ in diffusionweighted imaging (DWI) nor WMH via FLAIR. ${ }^{1,4}$ Recently, DTI

Received July 30, 2019; accepted after revision October 30

From the Departments of Neurology (C.I., Y.A.A.), Physical Therapy and Human Movement Sciences (C.I.), and Radiology (J.H.), Northwestern University, Chicago, Illinois; Department of Neurology (C.L.), University of Alabama at Birmingham, Birmingham, Alabama; and Department of Neurology (S.P.), University of Chicago Medical Center, Chicago, Illinois.

This study was funded through the Eleanor Wood Prince Grants Initiative of the Woman's Board of Northwestern University and the Davee Foundation.

Please address correspondence to Carson Ingo, PhD, Northwestern University, 645 N. Michigan Ave, Suite 850, Room 856, Chicago, IL 60611; e-mail: carson.ingo@northwestern.edu

http://dx.doi.org/10.3174/ajnr.A6357 of NAWM in patients with acute ischemic stroke has been used to determine the relationship between decreased whole-brain fractional anisotropy (FA) in NAWM and functional outcomes after ischemic stroke. ${ }^{12,13}$ There have been other studies that have investigated DTI measures $10-15$ days ${ }^{14}$ and $30+$ days ${ }^{15}$ following stroke. In this study, we performed an region of interest (ROI) DTI analysis of NAWM to determine the relationship between diffusivity metrics and the severity of motor impairment in the acute stages (within 72 hours of symptom onset) in patients with confirmed acute ischemic stroke compared with controls suspected of having ischemic stroke but without acute infarct.

\section{MATERIALS AND METHODS}

Participants and Clinical Assessment

The Northwestern University institutional review board approved the study. Patients older than 18 years of age with a confirmed 
Table 1: Demographics, clinical outcome measures, stroke risk factors, and acute infarct characteristics among those diagnosed with an acute ischemic stroke and those who were suspected of having an acute stroke but did not have an infarct (non-stroke)

\begin{tabular}{lccc}
\hline & Acute Stroke $(\boldsymbol{n}=52)$ & Nonstroke $(\boldsymbol{n}=36)$ & $\boldsymbol{P}$ \\
\hline Demographics & & & \\
Age (mean) (SD) (yr) & $69.2(17.4)$ & $62.2(17.7)$ & .068 \\
Women (No.) (\%) & $28(54.0 \%)$ & $19(52.8 \%)$ & .907 \\
Black (No.) (\%) & $17(32.7 \%)$ & $13(36.1 \%)$ & .715 \\
Clinical outcome measures & & & \\
Motricity Index (median) (IQR) & $77(44-92)$ & $\mathrm{NA}$ & - \\
NIH Stroke Scale (median) (IQR) & $4(2-13)$ & $\mathrm{NA}$ & - \\
Stroke risk factors & & & \\
Diabetes mellitus & $14(26.9 \%)$ & $7(19.4 \%)$ & .394 \\
Hypertension & $40(76.9 \%)$ & $24(66.7 \%)$ & .309 \\
Atrial fibrillation/flutter & $13(32.5 \%)$ & $8(22.2 \%)$ & .595 \\
Dyslipidemia & $27(51.9 \%)$ & $17(47.2 \%)$ & .652 \\
Cardiomyopathy & $14(26.9 \%)$ & $7(19.4 \%)$ & .271 \\
Smoking history & $17(32.7 \%)$ & $8(22.2 \%)$ & .612 \\
Infarct characteristics & & & \\
Median infarct volume (IQR) (mL) & $1.1(0.3-9.2)$ & $\mathrm{NA}$ & - \\
Infarct overlap with corticospinal tract & $36(69.2 \%)$ & $\mathrm{NA}$ & - \\
$\quad$ (No.) (\%) & & & \\
Side of infarct, right (No.) (\%) & $31(59.6 \%)$ & $\mathrm{NA}$ & - \\
Median white matter hyperintensity & $11.7(2.2-35.2)$ & $10.1(1.93-26.7)$ & .143 \\
$\quad$ volume (IQR) (mL) & & & \\
Stroke subtype & $12(23.1 \%)$ & $\mathrm{NA}$ & - \\
Cardioembolic & $11(21.2 \%)$ & $\mathrm{NA}$ & - \\
Small artery disease & $13(25.0 \%)$ & $\mathrm{NA}$ & - \\
Large atherosclerosis & $14(26.9 \%)$ & $\mathrm{NA}$ & - \\
Cryptogenic & $2(3.8 \%)$ & $\mathrm{NA}$ & - \\
Other & $10(19.2 \%)$ & $5(9.6 \%)$ & - \\
IV-tPA cases & & $\mathrm{NA}$ & \\
Thrombectomy rate & & & \\
\hline Note- & & & \\
\hline
\end{tabular}

Note:-NA indicates not applicable; IV-tPA, intravenous tissue-type plasminogen activator; NIH, National Institutes of Health.

acute ischemic stroke or suspected of having an acute stroke were enrolled. Written informed consent was obtained from the patient or his or her legally authorized representative for all participants. Patients with an acute stroke were defined as those with MR imaging evidence of acute infarct as identified on diffusion-weighted and apparent diffusion coefficient (ADC) images. Patients without an acute stroke (non-stroke) were defined as those who underwent MR imaging to rule out stroke if they had presented with any neurologic symptom within the 72 hours of medical center admission. The non-stroke patients did not have a diagnosis of an acute infarct, TIA, or history of stroke (ischemic or hemorrhagic) or TIA. Diagnosis was made by a board-certified vascular neurologist in each case. It was confirmed that participants did not have demyelinating disease or a prior stroke diagnosis before inclusion in this study. Clinical assessments with the Motricity Index (range, $0-100,100=$ no motor impairment), ${ }^{16}$ NIHSS (range, $0-42,0=$ no stroke symptoms), ${ }^{17}$ and MR imaging data were acquired in the participants within 72 hours of symptom onset. Patient demographics, premorbid characteristics, risk factors, and comorbidities were also collected as shown in Table 1 .

\section{DWI}

Axial diffusion-weighted images were acquired with the following parameters on a 3T MAGNETOM Skyra (Siemen, Erlangen, Germany) scanner: $\mathrm{TE}=95 \mathrm{~ms}$, TR $=10,000 \mathrm{~ms}$, flip angle $=90^{\circ}$, matrix size $=122 \times 122, \mathrm{FOV}=220 \times$ $220 \mathrm{~mm}^{2}$, section thickness $=2 \mathrm{~mm}$, voxel resolution $=1.8 \times 1.8 \times 2 \mathrm{~mm}^{3}$, slices $=74, \mathrm{~b}=0 \mathrm{~s} / \mathrm{mm}^{2}$ images $=3$, diffusion gradient directions $=30$, diffusion-weighting $=1000 \mathrm{~s} / \mathrm{mm}^{2}$.

\section{FLAIR Imaging}

Axial turbo spin-echo FLAIR images were acquired with the following parameters: $\mathrm{TE}=81 \mathrm{~ms}$, $\mathrm{TR}=9000 \mathrm{~ms}$, $\mathrm{TI}=2500 \mathrm{~ms}$, flip angle $=150^{\circ}$, matrix size $=320 \times 320, \mathrm{FOV}=220 \times$ $220 \mathrm{~mm}^{2}$, section thickness $=4 \mathrm{~mm}$, voxel resolution $=0.7 \times 0.7 \times 4.0$ $\mathrm{mm}^{3}$, slices $=30$.

\section{DWI Preprocessing and Tensor Fitting}

The diffusion-weighted images were first brain-extracted using the FSL Brain Extraction Tool. ${ }^{18}$ The data were then denoised using an estimate of the noise variance in the CSF signal intensity of the right ventricle. The data were corrected for motion and eddy currents by coregistering diffusion-weighted images to the image acquired with $b=0 \mathrm{~s} / \mathrm{mm}^{2}$ using the FMRIB Linear Image Registration Tool (https://fsl.fmrib.ox.ac.uk/fsl/ fslwiki/FLIRT). The motion-correction transformation matrix was applied to the diffusion gradient directions to rotate them according to the registration algorithm. The preprocessed diffusion-weighted data were fitted to a tensor on a voxelwise basis using dtifit in the FSL Diffusion Toolbox to produce estimates of FA, mean diffusivity (MD), radial diffusivity (RD), and axial diffusivity (AD).

\section{White Matter Hyperintensity and Infarct Segmentation}

FLAIR images were skull-stripped, denoised using a nonlocal means filter, ${ }^{19}$ and corrected for intensity nonuniformity. Cleaned images were input to the lesion prediction algorithm of the Lesion Segmentation Toolbox in SPM12 (https://www. applied-statistics.de/lst.html). Lesion probability maps were thresholded at greater than zero percentage probability to form initial masks and corrected using FreeSurfer and approved by a neurologist (C.L.). The brain-extracted FLAIR images were affine registered to the $b=0 \mathrm{~s} / \mathrm{mm}^{2}$ diffusion scan (3dAllineate; Analysis of Functional Neuro Images [AFNI]), and the resultant transform was applied to the corrected mask with nearest-neighbor interpolation to obtain diffusion-space WMH masks. The FLAIR images were brain-extracted and affine-registered to the $b=0 \mathrm{~s} / \mathrm{mm}^{2}$ diffusion scan using FLIRT in FSL. The same transformations were applied to the $\mathrm{WMH}$ mask images. 


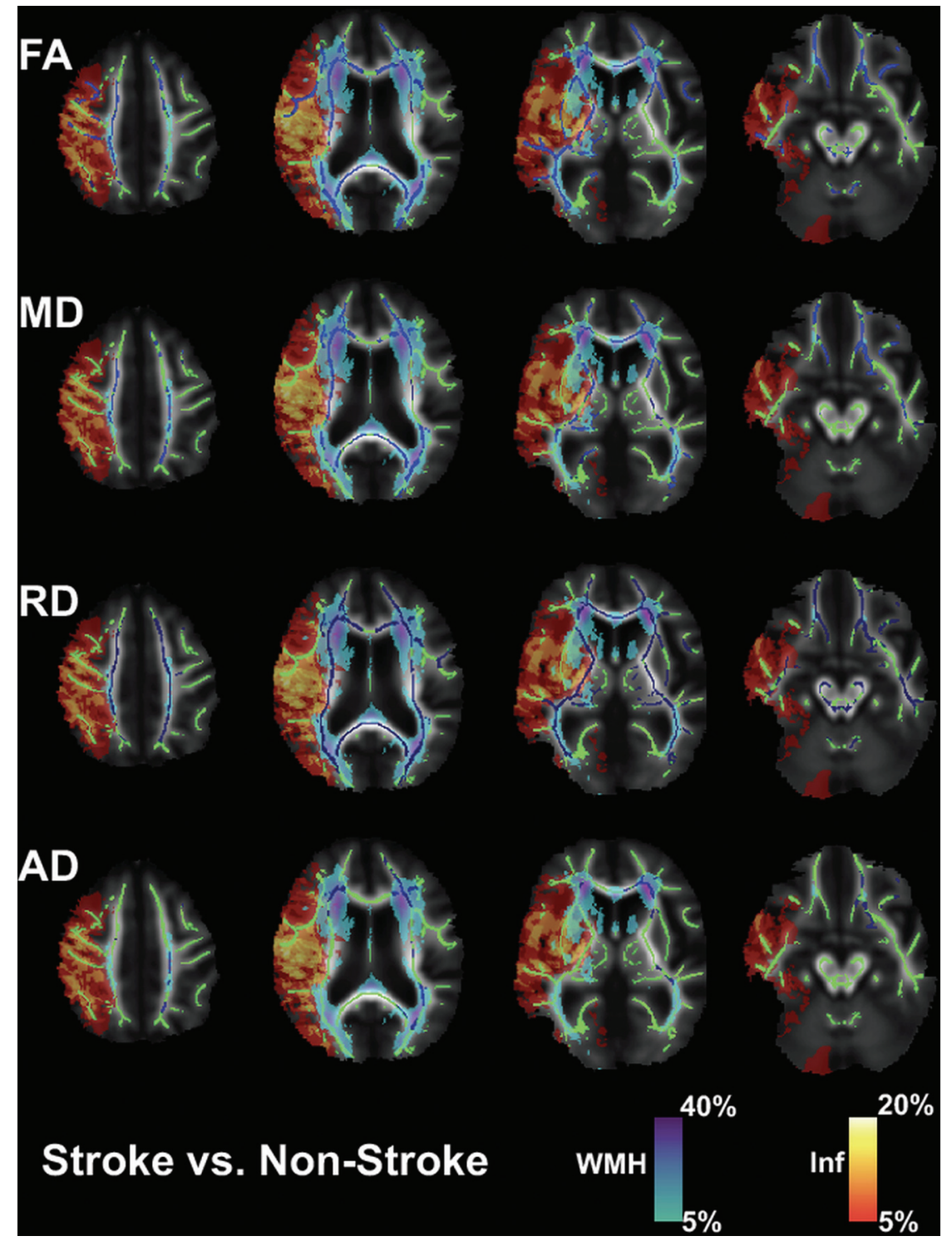

FIG 1. Group analysis for those diagnosed with an acute ischemic stroke and those who were suspected of having an acute stroke but did not have an infarct (non-stroke). Green represents the white matter skeleton for the group comparison. Blue represents significantly changed voxels $(P<.05)$ for FA, MD, RD, and AD: stroke FA < non-stroke FA, stroke MD > non-stroke $M D$, stroke $\mathrm{RD}>$ non-stroke $\mathrm{RD}$, and stroke $\mathrm{AD}>$ non-stroke $\mathrm{AD}$. The cool map represents the WMH probability map from FLAIR images. The heat map represents the infarct (Inf) probability map from $M D$ images. Statistical analyses were adjusted for age as a covariate and corrected for multiple comparisons using permutation-based testing.

The infarct voxels were segmented using a threshold approach in which values were less than $0.68 \times 10^{-3} \mathrm{~mm}^{2} / \mathrm{s}$ from the MD images to produce the infarct masks. ${ }^{20}$ These were manually corrected using FreeSurfer and approved by a neurologist (C.L.).

\section{Statistical Analysis}

Tract-Based Spatial Statistics (TBSS) was performed in FSL. ${ }^{21}$ For those individuals with infarcts in the left hemisphere, FA maps were flipped so that all subjects had infarcts in the right hemisphere. FA maps were first linearly and then nonlinearly registered to the FMRIB58_FA in the standard space of the Montreal Neurological Institute. A mean FA image was then created from all individual FA images and was used to generate a common group skeleton. A threshold was applied at 0.2 to minimize potential white matter/gray matter partial volume effects. Each FA image was projected onto the common group skeleton for subsequent statistical analysis. For those individuals with infarcts in the left hemisphere, $\mathrm{MD}, \mathrm{RD}, \mathrm{AD}$, infarct, and $\mathrm{WMH}$ maps were flipped so that all subjects had infarcts in the right hemisphere. The same transformations that were applied to the FA maps were also applied to the $\mathrm{MD}, \mathrm{RD}, \mathrm{AD}$, infarct, and $\mathrm{WMH}$ maps. The infarct (heat) and WMH (cool) maps are shown in Figs 1 and 2 and are presented as a percentage of those individuals who had instances of infarct or white matter hyperintensity voxels with respect to the total acute stroke group. The Johns Hopkins University ICBM-DTI-81 white matter labels and tractography atlases were used to identify the white matter ROIs that contained the significantly changed voxels. $^{22-24}$

Test 1. To investigate potential differences in diffusivity metrics in those diagnosed with an acute ischemic stroke and those who were suspected of having an acute stroke but did not have an infarct (non-stroke), a 2group unpaired $t$ test was performed with age as a covariate. In order to correct for multiple comparisons, permutation testing $(n=500)$ was performed using the randomise and threshold-free cluster enhancement (TFCE) functions in FSL. ${ }^{25}$

Test 2. To test for possible significant interactions between the diffusivity metrics and each individual risk factor (diabetes mellitus, hypertension, atrial fibrillation/flutter, dyslipidemia, cardiomyopathy, and smoking history) for those who were diagnosed with an acute stroke, a partial correlation analysis was performed with age as a covariate. In order to correct for multiple comparisons, permutation testing $(n=500)$ was performed. ${ }^{25}$

Test 3. To investigate potential associations between the diffusivity metrics and the Motricity Index for those who were diagnosed with an acute stroke, a partial correlation analysis was performed with age, total infarct volume, and infarct volume overlap with the corticospinal tract as covariates. In order to correct for multiple comparisons, permutation testing $(n=500)$ was performed. ${ }^{25}$ 


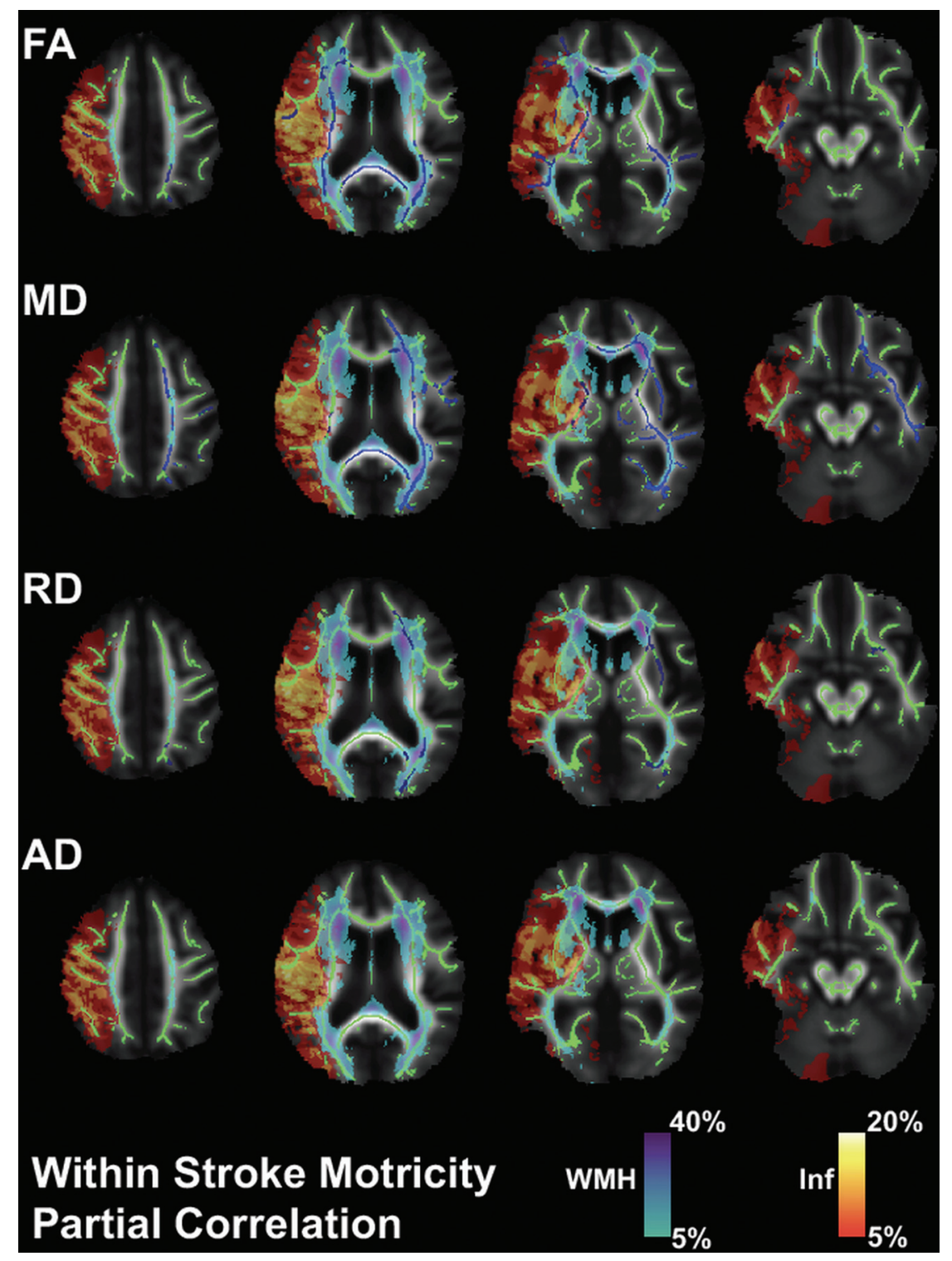

FIG 2. Partial correlation analysis for those diagnosed with an acute ischemic stroke. Green represents the white matter skeleton for the imaging measures versus the Motricity Index (MI) correlation. Blue represents significantly changed voxels $(P<.05)$ for $F A, M D, R D$, and $A D$ : FA positively associated with MI, MD negatively associated with MI, RD negatively associated with $M I$, and AD not significantly associated with MI. The cool map represents the WMH probability map from FLAIR images. The heat map represents the infarct (Inf) probability map from MD images. Statistical analyses were adjusted for covariates of age, total infarct volume, and infarct volume overlap with the corticospinal tract and then were corrected for multiple comparisons using permutation-based testing.

\section{RESULTS}

As shown in Table 1, the final analysis included 52 patients with acute ischemic stroke and 36 without stroke (non-stroke group) who were enrolled consecutively. In the patients with stroke, the mean age was $69.2 \pm 17.4$ years, 28 were women $(54 \%)$, and 17 were black (32.7\%). There were no statistical differences in these baseline characteristics between the stroke and non-stroke groups. Within the stroke group, the median Motricity Index was 77 (interquartile range [IQR], 44-92) and the median NIHSS score was 4 (IQR, 2-13). The median infarct volume in the stroke group was $1.1 \mathrm{~mL}$ (IQR, 0.3-9.2 mL) with predominance in the right hemisphere in 31 (59.6\%), and 36 infarcts (69.2\%) had overlap with the corticospinal tract.

\section{Acute Ischemic Stroke versus Non-stroke Controls}

As shown in Fig 1, the NAWM voxels (green) that exclude infarct (heat map) and white matter hyperintensity (cool map) probability masks were tested for group differences in $\mathrm{FA}, \mathrm{MD}, \mathrm{RD}$, and $\mathrm{AD}$ between the acute stroke group and the nonstroke group. There were widespread decreases in FA (blue, panel 1) and increases in MD and RD (blue, panels 2 and 3) for the acute stroke group compared with the nonstroke group. There were minimal increases in $\mathrm{AD}$ (blue, panel 4) for the acute stroke group compared with the non-stroke group; however, most of these voxels overlapped with the WMH probability map.

\section{Acute Stroke Risk Factor Testing}

Each individual risk factor (diabetes mellitus, hypertension, atrial fibrillation/flutter, dyslipidemia, cardiomyopathy, and smoking history) for those who were diagnosed with an acute stroke was tested for significant interactions with $\mathrm{FA}, \mathrm{MD}, \mathrm{RD}$, and AD. None of the risk factors had any significant interaction with the diffusivity metric voxels. Additionally, potential asymmetry effects ${ }^{26}$ in the diffusion metrics between hemispheres were investigated. The individuals who were flipped were compared with the non-flipped individuals in a 2-group unpaired $t$ test with Motricity Index, age, total infarct volume, and infarct volume overlap with the corticospinal tract as covariates to isolate potential effects of asymmetry. There were no significant voxels.

\section{Acute Stroke Testing for Motor Impairment Measured by the Acute Motricity Index}

As shown in Fig 2, in stroke, for the NAWM voxels tested for partial correlation with the Motricity Index, 12.1\% FA, 28.9\% $\mathrm{MD}$, and 3.8\% RD voxels were significant (blue) with respect to the NAWM TBSS skeleton (green). There was a positive association between FA and the Motricity Index and a negative association between $\mathrm{MD} / \mathrm{RD}$ and the Motricity Index. There were no 
Table 2: FA versus the Motricity Index adjusted for covariates of age, total infarct volume, and infarct volume overlap with the corticospinal tract ${ }^{\mathrm{a}}$

\begin{tabular}{|c|c|c|c|c|}
\hline & $\begin{array}{l}\text { No. of Significant } \\
\text { Voxels }\end{array}$ & $\begin{array}{l}\text { Significant } \\
\text { Voxels (\%) }\end{array}$ & $r$ & $P$ \\
\hline Anterior corona radiata, $\mathrm{R}$ & 69 & $4.4 \%$ & 0.379 & .027 \\
\hline Anterior limb of internal capsule, $\mathrm{R}$ & 383 & $55.1 \%$ & 0.396 & .025 \\
\hline Body of corpus callosum & 23 & $2.4 \%$ & 0.275 & .032 \\
\hline Cerebral peduncle, $\mathrm{R}$ & 47 & $10.1 \%$ & 0.285 & .030 \\
\hline Cingulum (cingulate gyrus), L & 22 & $3.8 \%$ & 0.252 & .032 \\
\hline Cingulum (cingulate gyrus), $\mathrm{R}$ & 41 & $6.8 \%$ & 0.297 & .032 \\
\hline External capsule, $\mathrm{L}$ & 86 & $11.0 \%$ & 0.311 & .030 \\
\hline External capsule, $\mathrm{R}$ & 129 & $19.5 \%$ & 0.392 & .033 \\
\hline Genu of corpus callosum & 17 & $5.6 \%$ & 0.237 & .039 \\
\hline Posterior limb of internal capsule, $\mathrm{R}$ & 419 & $73.6 \%$ & 0.434 & .023 \\
\hline Posterior thalamic radiation, $\mathrm{L}$ & 22 & $4.0 \%$ & 0.296 & .035 \\
\hline $\begin{array}{l}\text { Retrolenticular part of internal } \\
\text { capsule, } L\end{array}$ & 20 & $14.0 \%$ & 0.269 & .036 \\
\hline $\begin{array}{l}\text { Retrolenticular part of internal } \\
\text { capsule, } R\end{array}$ & 87 & $31.3 \%$ & 0.398 & .027 \\
\hline Sagittal stratum, L & 131 & $37.6 \%$ & 0.345 & .031 \\
\hline Sagittal stratum, $\mathrm{R}$ & 128 & $29.6 \%$ & 0.361 & .031 \\
\hline Splenium of corpus callosum & 1068 & $65.2 \%$ & 0.443 & .023 \\
\hline Superior corona radiata, $\mathrm{L}$ & 40 & $3.1 \%$ & 0.371 & .034 \\
\hline Superior corona radiata, $\mathrm{R}$ & 188 & $13.8 \%$ & 0.563 & .021 \\
\hline Superior longitudinal fasciculus, $\mathrm{L}$ & 126 & $8.7 \%$ & 0.307 & .039 \\
\hline Superior longitudinal fasciculus, $R$ & 68 & $5.0 \%$ & 0.536 & .033 \\
\hline Uncinate fasciculus, $\mathrm{R}$ & 23 & $30.3 \%$ & 0.451 & .033 \\
\hline
\end{tabular}

Note:- $L$ indicates left; $R$, right.

a The reported $P$ value is corrected for multiple comparisons using permutation-based testing. Voxels are reported from NAWM ROls that exclude infarcts and white matter hyperintensities visible from FLAIR images. The right hemisphere represents the infarcted hemisphere.

significant $\mathrm{AD}$ voxels. The right hemisphere represents the infarcted hemisphere (InfHem).

Table 2 shows the ROI breakdown of the significantly changed voxel overlay with the Johns Hopkins University ICBMDTI-81 white matter labels for the FA-Motricity Index partial correlation. The splenium of the corpus callosum (1068, $r=$ $0.443, P=.023$ ) had the most significant number of voxels, followed by the right (InfHem) posterior limb of the internal capsule (419, $r=0.434, P=.023$ ), the right (InfHem) anterior limb of the internal capsule (383, $r=0.396, P=.025)$, the right (InfHem) superior corona radiata $(188, r=0.563, P=.021)$, and the left/ right sagittal stratum (131, $r=0.345, P=.031 / 128, r=0.361$, $P=.031$ ). The right (InfHem) posterior limb of the internal capsule $(73.6 \%, r=0.434, P=.023)$ had the largest percentage of significant ROI voxels, followed by the splenium of the corpus callosum (65.2\%, $r=0.443, P=.023$ ), the right (InfHem) anterior limb of the internal capsule $(55.1 \%, r=0.396, P=.025)$, the left/ right sagittal stratum $(37.6 \%, r=0.345, P=.031 / 29.6 \%, r=0.361$, $P=.031$ ), the right (InfHem) retrolenticular part of the internal capsule $(31.3 \%, r=0.398, P=.027)$, and the right (InfHem) uncinate fasciculus $(30.3 \%, r=0.451, P=.033)$.

Table 3 shows the ROI breakdown of the significantly changed voxel overlay with the Johns Hopkins University white matter labels for the MD-Motricity Index partial correlation. The splenium of the corpus callosum $(947, r=-0.450, P=.012$ ) had the most significant number of voxels, followed by the left superior longitudinal fasciculus (561, $r=-0.448, P=.021$ ), the left external capsule (544, $r=-0.492, P=.017)$, the right (InfHem) anterior limb of internal capsule (282, $r=-0.481, P=.047)$, the left posterior limb of the internal capsule $(274, r=-0.400, P=.014)$, and the left sagittal stratum $(220, r=-0.457$, $P=.015)$. The left cingulum (hippocampus) (91.2\%, $r=-0.385, P=.021)$ had the largest percentage of significant ROI voxels, followed by the left retrolenticular part of internal capsule (87.4\%, $r=-0.368, P=.014)$, the left posterior limb of the internal capsule (74.7\%, $r=-0.400, P=.014)$, the left external capsule $(69.6 \%, r=-0.492$, $P=.017)$, the left uncinate fasciculus (65.0\%, $r=-0.428, P=.011)$, the left sagittal stratum $(63.2 \%, r=-0.457$, $P=.015)$, and the splenium of the corpus callosum (57.8\%, $r=-0.450, P=.012)$.

Table 4 shows the ROI breakdown of the significantly changed voxel overlay with the Johns Hopkins University white matter labels for the $\mathrm{RD}$ Motricity Index partial correlation. The left external capsule $(155, r=-0.409$, $P=.018$ ) had the most significant number of voxels, followed by the left posterior thalamic radiation $(56, r=-0.298$, $P=.029)$, and the splenium of the corpus callosum (44, $r=-0.273, P=.032$ ). The left external capsule (19.8\%, $r=-0.409, P=.018)$ had the largest percentage of significant ROI voxels, followed by the left posterior thalamic radiation (10.1\%, $r=-0.298, P=.029)$.

There were no significantly changed voxels for the ADMotricity Index partial correlation.

\section{DISCUSSION \\ Occurrence of Acute Stroke in Relation to Normal- Appearing White Matter Integrity}

As evidenced by the FA, MD, and RD results for the group test between acute ischemic stroke and non-stroke participants, there appears to be a decrease in integrity for normal-appearing white matter surrounding not only the infarcted hemisphere, which was predominately the MCA territory, but also in the contralateral hemisphere. Previous work has shown that whole-brain integrity of normal-appearing white matter is associated with functional motor outcomes following ischemic acute stroke. ${ }^{12,13}$ A recent DTI study analyzed cognitive impairment 10-14 days following stroke in comparison with a control group, but there were no delineations between abnormal and normal-appearing white matter. ${ }^{14}$ However, there has been a lack of investigations into the baseline NAWM integrity in those who have been imaged within 72 hours of stroke onset compared with those without stroke. The new evidence presented in this study suggests that baseline normal-appearing white matter microstructural integrity in the infarcted hemisphere, intrahemisphere, and contralateral hemisphere may potentially be an additional risk factor that determines the susceptibility to stroke occurrence, which 
Table 3: MD versus the Motricity Index adjusted for covariates of age, total infarct volume, and infarct volume overlap with the corticospinal tract ${ }^{\mathrm{a}}$

\begin{tabular}{lcccc}
\hline & $\begin{array}{c}\text { No. of Significant } \\
\text { Voxels }\end{array}$ & $\begin{array}{c}\text { Significant } \\
\text { Voxels (\%) }\end{array}$ & $\boldsymbol{r}$ & $\boldsymbol{P}$ \\
\hline Anterior corona radiata, L & 29 & $2.1 \%$ & -0.390 & .021 \\
Anterior limb of internal capsule, R & 282 & $40.6 \%$ & -0.481 & .047 \\
Body of corpus callosum & 21 & $2.2 \%$ & -0.259 & .031 \\
Cerebral peduncle, L & 136 & $26.7 \%$ & -0.388 & .020 \\
Cerebral peduncle, R & 69 & $14.8 \%$ & -0.378 & .047 \\
Cingulum (cingulate gyrus), L & 19 & $3.3 \%$ & -0.313 & .030 \\
Cingulum (hippocampus), L & 124 & $91.2 \%$ & -0.385 & .021 \\
External capsule, L & 544 & $69.6 \%$ & -0.492 & .017 \\
External capsule, R & 21 & $3.2 \%$ & -0.390 & .048 \\
Genu of corpus callosum & 106 & $34.6 \%$ & -0.336 & .037 \\
Posterior limb of internal capsule, L & 274 & $74.7 \%$ & -0.400 & .014 \\
Posterior limb of internal capsule, R & 158 & $27.8 \%$ & -0.412 & .048 \\
Posterior thalamic radiation, L & 183 & $33.1 \%$ & -0.465 & .010 \\
Retrolenticular part of internal & 125 & $87.4 \%$ & -0.368 & .014 \\
$\quad$ capsule, L & & & & \\
Retrolenticular part of internal & 21 & $7.6 \%$ & -0.353 & .048 \\
$\quad$ capsule, R & & & & \\
Sagittal stratum, L & 220 & $63.2 \%$ & -0.457 & .015 \\
Splenium of corpus callosum & 947 & $57.8 \%$ & -0.450 & .012 \\
Superior corona radiata, L & 194 & $15.1 \%$ & -0.350 & .017 \\
Superior longitudinal fasciculus, L & 561 & $38.6 \%$ & -0.448 & .021 \\
Uncinate fasciculus, L & 39 & $65.0 \%$ & -0.428 & .011 \\
\hline Not & & & &
\end{tabular}

Note:- $L$ indicates left; $R$, right.

${ }^{a}$ The reported $P$ value is corrected for multiple comparisons using permutation-based testing. Voxels are reported from NAWM ROIs that exclude infarcts and white matter hyperintensities visible from FLAIR images.

Table 4: RD versus Motricity Index adjusted for covariates of age, total infarct volume, and infarct volume overlap with the corticospinal tract ${ }^{\mathrm{a}}$

\begin{tabular}{lcccc}
\hline & $\begin{array}{c}\text { Total No. of } \\
\text { Significant Voxels }\end{array}$ & $\begin{array}{c}\text { Total Percentage of } \\
\text { Significant Voxels }\end{array}$ & $\boldsymbol{r}$ & $\boldsymbol{P}$ \\
\hline Cerebral peduncle, $\mathrm{L}$ & 17 & $3.3 \%$ & -0.269 & .030 \\
External capsule, L & 155 & $19.8 \%$ & -0.409 & .018 \\
Posterior limb of internal & 19 & $5.2 \%$ & -0.315 & .028 \\
$\quad$ capsule, L & & & & \\
Posterior thalamic radiation, $\mathrm{L}$ & 56 & $10.1 \%$ & -0.298 & .029 \\
Splenium of corpus callosum & 44 & $2.7 \%$ & -0.273 & .032 \\
\hline
\end{tabular}

Note:- $L$ indicates left

a The reported $P$ value is corrected for multiple comparisons using permutation-based testing. Voxels are reported from NAWM ROIs that exclude infarcts and white matter hyperintensities visible from FLAIR images. limb/retrolenticular part of the internal capsule, superior longitudinal fasciculus, and cingulum (hippocampus) with the partial correlation to initial Motricity Index score for motor impairment, there appears to be a decrease in integrity for normalappearing white matter in the intrahemisphere and contralateral hemisphere that relates to the initial severity of the injury.

\section{Relationship of Normal- Appearing White Matter Microstructural Integrity and Traditional Imaging Markers}

This study considered both the functional locations and volumes of traditional markers of neural injury and burden via infarcted and WMH tissue as covariates to isolate, as well as possible, the relationship between NAWM microstructural integrity and the severity of motor impairment in acute ischemic stroke. Because there were statistically significant correlations, particularly with $\mathrm{FA} / \mathrm{MD}$ and the Motricity Index, in motor ROIs in each of the infarcted hemispheres, intrahemisphere, and contralateral hemisphere, these results suggest that the health of NAWM is an additional important variable along with traditional metrics of infarct volume, infarct location, and WMH burden ${ }^{1-7}$ to better understand the severity and extent of functional impairment. builds on previous reports of overt $\mathrm{WMH}$ as a risk factor. ${ }^{27}$ However, it is still possible that widespread changes in NAWM diffusivity metrics in patients with acute ischemic stroke compared with non-stroke are sequelae of the acute infarct.

\section{Severity of Initial Motor Impairment Due to Acute Stroke and Infarcted Hemisphere Normal-Appearing White Matter Integrity}

As evidenced by the FA and MD results for the ipsilateral anterior/posterior limb of the internal capsule with the partial correlation to the initial Motricity Index score for motor impairment, there appears to be a decrease in integrity for normal-appearing white matter in the infarcted hemisphere, which relates to the initial severity of the injury.

Severity of Initial Motor Impairment Due to Acute Stroke and Intrahemisphere/Contralateral Hemisphere NormalAppearing White Matter Microstructural Integrity

As evidenced by the FA and MD results for the splenium of the corpus callosum and the contralateral external capsule, posterior

\section{Significant MD Results and Relationship to the Apparent Diffusion Coefficient (ADC)}

Because the MD (28.9\% of voxels) results showed more significance in the statistical testing of the white matter skeleton in comparison with FA (12.1\% of voxels), this study is optimistic for larger studies being leveraged using typical clinical stroke MR imaging protocol DWI scans that sample only 3 diffusion directions instead of the 30 directions used in this study. MD is a metric akin to what is typically referred to as the ADC, which is calculated from a 3-diffusion-direction DWI scan. ${ }^{28}$ Because a minimum of 6 diffusion directions are needed to compute the diffusion tensor, ${ }^{29}$ it is not possible to calculate, for example, FA. However, because MD and ADC are both average diffusivity measures, it is possible that a 3-direction DWI scan could provide similar insight into NAWM following acute ischemic stroke.

\section{Clinical Implications and Impact}

The results presented in the current study provide the clinical potential to improve on previous attempts to develop multimodal algorithms and models ${ }^{30}$ to predict the likelihood and capacity 
for recovery following stroke. One study has shown that the ability to detect the preservation of the cortical spinal tract based on early DTI measures is an important parameter that predicts motor recovery and those who would benefit from targeted intervention and therapy strategies. ${ }^{30}$ It is possible that not only early estimates of available motor resources in the infarcted hemisphere are important, but early estimates of intrahemisphere and contralateral hemisphere normal-appearing white matter, as evidenced in the current study, are also relevant to better understand the potential for extent of motor recovery. These results may lead to further inquiries as to whether changes in normal-appearing white matter are present before or are a result of the acute injury and whether the decreased integrity in the intrahemisphere and contra lateral hemisphere is chronic in nature.

\section{Limitations}

The Johns Hopkins University white matter label atlas ${ }^{22-24}$ covers only a subset of the white matter skeleton used for statistical analysis in this study. Therefore, significant voxels are not represented in the ROI analysis presented in Tables 2-4. Because this study is focused on both the acute imaging analysis and clinical assessment of motor function in ischemic stroke within 72 hours of symptom onset, future work is needed to perform follow-up imaging analysis and clinical assessment at 3-6 months to determine the possibility of long-term motor recovery with respect to the viability of the NAWM microstructure. Vascular neurologists in this study did not record further clinical diagnosis of the etiology of neurologic symptoms for the nonstroke patient group once they were determined to not have strokes. While the present study focused on milder cases of stroke, it has been shown from a public health standpoint that those with minor strokes (NIHSS $<3$ ) are the most commonly reported group of patients with strokes in the United States ${ }^{31}$ which further emphasizes that there are meaningful acute imaging parameters associated with motor impairment, even in patients with relatively mild severity. While potential asymmetry effects have been reported previously in the diffusion metrics between hemispheres ${ }^{26}$ and they were found in this study to not produce significant results between flipped versus nonflipped images, it is possible that potential hemispheric asymmetry could contribute to the imaging results shown in Figs 1 and 2.

\section{CONCLUSIONS}

The microstructural integrity of NAWM, as measured by diffusion tensor parameters, is a significant parameter to not only identify microstructural differences between those individuals with acute ischemic stroke compared with those who did not have an infarct but also to correlate with the severity of acute motor impairment. In this study, the NAWM ROIs that were most sensitive to the Motricity Index were the anterior/posterior limb of the internal capsule in the infarcted hemisphere and the splenium of the corpus callosum, external capsule, posterior $\mathrm{limb} /$ retrolenticular part of the internal capsule, superior longitudinal fasciculus, and cingulum (hippocampus) of the intrahemisphere/contralateral hemisphere. Future studies could include the 3- to 6-month follow-up of the morphology of these imaging measures in relation to clinical assessment, recovery, and rehabilitation of motor function.
Disclosures: Carson Ingo-RELATED: Grant: The data collection of this study was funded through the Eleanor Wood Prince Grants Initiative of the Woman's Board of Northwestern University and the Davee Foundation.* Chen LinRELATED: Grant: Woman's Board of Northwestern Memorial Hospital, Comments: covered imaging scans.* Yurany A. Arevalo—RELATED: Grant: the Eleanor Wood Prince Grants Initiative of the Woman's Board of Northwestern University and the Davee Foundation.* Shyam Prabhakaran-RELATED: Grant: Women's Board Foundation Grant*; UNRELATED: Grants/Grants Pending: National Institutes of Health/National Institute of Neurological Disorders and Stroke, Agency for Healthcare Research and Quality*; Royalties: UpToDate.* *Money paid to institution.

\section{REFERENCES}

1. Henninger N, Lin E, Haussen DC, et al. Leukoaraiosis and sex predict the hyperacute ischemic core volume. Stroke 2013;44:61-67 CrossRef Medline

2. Ay H, Arsava EM, Rosand J, et al. Severity of leukoaraiosis and susceptibility to infarct growth in acute stroke. Stroke 2008;39:1409-13 CrossRef Medline

3. Arsava EM, Rahman R, Rosand J, et al. Severity of leukoaraiosis correlates with clinical outcome after ischemic stroke. Neurology 2009;72:1403-10 CrossRef Medline

4. Henninger N, Lin E, Baker SP, et al. Leukoaraiosis predicts poor 90-day outcome after acute large cerebral artery occlusion. Cerebrovasc Dis 2012;33:525-31 CrossRef Medline

5. Kissela B, Lindsell CJ, Kleindorfer D, et al. Clinical prediction of functional outcome after ischemic stroke: the surprising importance of periventricular white matter disease and race. Stroke 2009;40:530-56 CrossRef Medline

6. Schirmer MD, Dalca AV, Sridharan R, et al. White matter hyperintensity quantification in large-scale clinical acute ischemic stroke cohorts: the MRI-GENIE study. Neuroimage Clin 2019;23:101884 CrossRef Medline

7. Khan M, Heiser H, Bernicchi N, et al. Leukoaraiosis predicts shortterm cognitive but not motor recovery in ischemic stroke patients during rehabilitation. J Stroke Cerebrovasc Dis 2019;28:1597-603 CrossRef Medline

8. de Groot M, Verhaaren BFJ, de Boer R, et al. Changes in normalappearing white matter precede development of white matter lesions. Stroke 2013;44:1037-42 CrossRef Medline

9. Vernooij MW, Ikram MA, Vrooman HA, et al. White matter microstructural integrity and cognitive function in a general elderly population. Arch Gen Psychiatry 2009;66:545-53 CrossRef Medline

10. Sorensen AG, Wu O, Copen WA, et al. Human acute cerebral ischemia: detection of changes in water diffusion anisotropy by using MR imaging. Radiology 1999;212:785-92 CrossRef Medline

11. Dacosta-Aguayo R, Graña $M$, Fernández-Andújar $M$, et al. Structural integrity of the contralesional hemisphere predicts cognitive impairment in ischemic stroke at three months. PLoS One 2014;9:e86119 CrossRef Medline

12. Etherton $\mathrm{MR}, \mathrm{Wu} \mathrm{O}$, Cougo $\mathrm{P}$, et al. Integrity of normal-appearing white matter and functional outcomes after acute ischemic stroke. Neurology 2017;88:1701-08 CrossRef Medline

13. Etherton $\mathrm{MR}, \mathrm{Wu} \mathrm{O}$, Giese $\mathrm{AK}$, et al. White matter integrity and early outcomes after acute ischemic stroke. Transl Stroke Res 2019;10:630-38 CrossRef Medline

14. Zuo LJ, Li ZX, Zhu RY, et al. The relationship between cerebral white matter integrity and cognitive function in mild stroke with basal ganglia region infarcts. Sci Rep 2018;8:8422 CrossRef Medline

15. Zamboni G, Griffanti L, Mazzucco S, et al. Age-dependent association of white matter abnormality with cognition after TIA or minor stroke. Neurology 2019;93:e272-82 CrossRef Medline

16. Demeurisse G, Demol O, Robaye E. Motor evaluation in vascular hemiplegia. Eur Neurol 1980;19:382-89 CrossRef Medline

17. Brott T, Adams HP Jr, Olinger CP, et al. Measurements of acute cerebral infarction: a clinical examination scale. Stroke 1989;20:864-70 CrossRef Medline 
18. Smith SM, Jenkinson M, Woolrich MW, et al. Advances in functional and structural MR image analysis and implementation as FSL. Neuroimage 2004;23: S208-19 CrossRef Medline

19. Manjon JV, Coupé P, Martí-Bonmatí L, et al. Adaptive non-local means denoising of MR images with spatially varying noise levels. J Magn Reson Imaging 2010;31:192-203 CrossRef Medline

20. Lin C, Sangha R, Lee J, et al. Infarct location is associated with quality of life after mild ischemic stroke. Stroke 2018;13:824-31 CrossRef Medline

21. Smith SM, Jenkinson M, Johansen-Berg H, et al. Tract-based spatial statistics: voxelwise analysis of multi-subject diffusion data. Neuroimage 2006;31:1487-505 CrossRef Medline

22. Mori S, Oishi K, Jiang H, et al. Stereotaxic white matter atlas based on diffusion tensor imaging in an ICBM template. Neuroimage 2008;40:570-82 CrossRef Medline

23. Oishi K, Zilles K, Amunts K, et al. Human brain white matter atlas: identification and assignment of common anatomical structures in superficial white matter. Neuroimage 2008;43:447-57 CrossRef Medline

24. Hua K, Zhang J, Wakana S, et al. Tract probability maps in stereotaxic spaces: analyses of white matter anatomy and tract-specific quantification. Neuroimage 2008;39:336-47 CrossRef Medline
25. Smith SM, Nichols TE. Threshold-free cluster enhancement: addressing problems of smoothing, threshold dependence and localisation in cluster inference. Neuroimage 2009;44:83-98 CrossRef Medline

26. Gong G, Jiang T, Zhu C, et al. Asymmetry analysis of cingulum based on scale-invariant parameterization by diffusion tensor imaging. Hum Brain Mapp 2005;24:92-98 CrossRef Medline

27. Debette $S$, Markus HS. The clinical importance of white matter hyperintensities on brain magnetic resonance imaging: systematic review and meta-analysis. BMJ 2010;341:c3666 CrossRef Medline

28. Basser PJ, Mattiello J, LeBihan D. Estimation of the effective self-diffusion tensor from the NMR spin echo. J Magn Reson B 1994; 103:247-54 CrossRef Medline

29. Basser PJ, Mattiello J, LeBihan D. MR diffusion tensor spectroscopy and imaging. Biophys J 1994;66:259-67 CrossRef Medline

30. Stinear CM, Byblow WD, Ackerley SJ, et al. A biomarker-based algorithm for predicting upper limb function after stroke. Ann Clin Transl Neurol 2017;4:811-20 CrossRef Medline

31. Reeves M, Khoury J, Alwell K, et al. Distribution of National Institutes of Health stroke scale in the Cincinnati/Northern Kentucky stroke study. Stroke 2013;44: 3211-13 CrossRef Medline 